\title{
Forakam: Imaging photothermal radiometry
}

\author{
by P. Menner*, J. Koch*, D. Saal**, P. Mayr** \\ * edevis GmbH, Handwerkstr. 55, 70565 Stuttgart, Germany, philipp.menner@edevis.de \\ ** Institute for Laser Technology in Medicine and Measurement Technique, Helmholtzstraße 12, 89081 UIm
}

\begin{abstract}
Photothermal radiometry is being used e.g. for characterization of changes in the microstructure of metals. It is non-destructive, non-contact and much faster than a metallographic analysis. However, this method uses only one detector element, therefore the inspection of areas requires time-consuming scanning. In this paper, we will show that infrared cameras can be used for detection as well. With an exact synchronization between the excitation laser and the IR image grabbing, reproducible phase contrast values can be obtained, thus converting photothermal radiometry into an imaging method. This is demonstrated on samples with different case hardening depths and grinding burn respectively.
\end{abstract}

\section{Introduction}

Advances in infrared camera technology have led not only to a higher lateral resolution, but also to higher framerates. So, not only thermally 'slow' materials like polymers and composites can be inspected by lockin thermography, but also the imaging testing of metal becomes possible. The major challenge is the perfect synchronization between the excitation laser and the camera stream. When inspecting composites, the heat flow effects are so slow that tests may even be performed without any synchronization between excitation and image grabbing. However, when small changes in the metallic microstructure shall be characterized, the phase contrast is just a few degrees, and random phase drifts and offsets caused by jitter and other delays can easily have a larger influence to the phase signal than the actual heat flow effects. During the Forakam project, we could improve the standard laser lockin thermography system LTvis to a level where photothermal radiometry can be performed reproducibly in an imaging way. Recently, an automotive OEM already proved this system to be able to perform valid measurements of thermally sprayed layers.

\section{Theoretical context}

Photothermal radiometry has been known for being able to measure layer thickness for a long time [1, 2]. The analytical solution for the phase angle of a thermal wave which diffuses though a single layer (under the boundary conditions of one-dimensional heat flux and the disregard of multiple reflection) is given by eq. (1) [3]:

$$
\varphi=\arctan \left(\frac{-2 R_{23} e^{-2 d \sqrt{\pi f / \alpha}} \sin 2 d \sqrt{\pi f / \alpha}}{1-\left(R_{23} e^{-2 d \sqrt{\pi f / \alpha}}\right)^{2}}\right)-\frac{\pi}{4}
$$

with $d$ being the thickness of the layer, $R_{23}$ the thermal reflection coefficient between layer and substrate, $\alpha$ the thermal diffusivity of the layer and $f$ the excitation frequency.

The Forakam system is able to obtain quantitative phase values e.g. for layers for a wide range of laser modulation frequencies. Figure 1 shows the measured data of a metallic layer on a cylinder surface and a fit according to equation (1), with the fit parameters being the layer thickness $d$ and the reflection coefficient $R_{23}$.

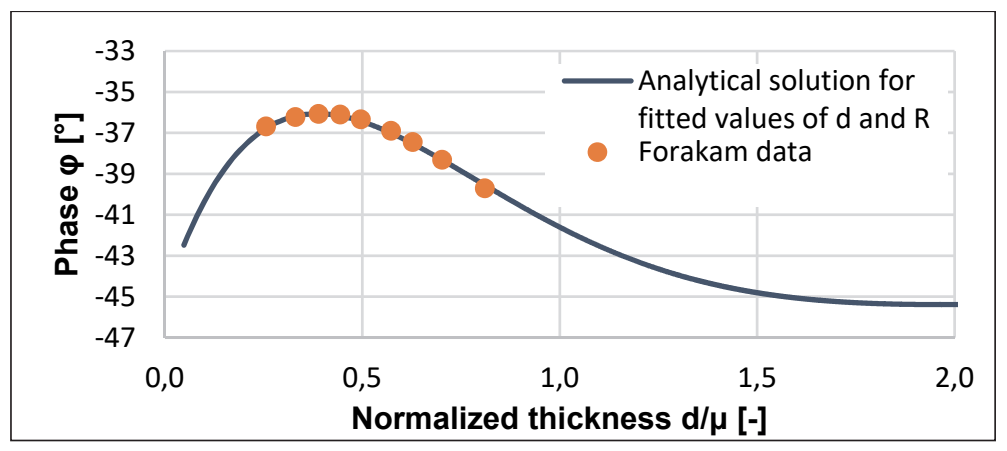

Fig. 1: Comparison of Forakam data and analytical $1 D$ solution for fitted values of $d$ and $R_{23}$. 
A detailed measurement system analysis - regarding amongst other things the repeatability and the reproducibility - showed that the Forakam system is capable of performing imaging photothermal radiometric analysis on an industrial level [4]. The next steps will include research whether the reflection coefficient can be used as a measure of the quality of the layer structure (regarding porosity etc.).

\section{Experimental setup}

The main components of the Forakam system are a $250 \mathrm{~W}$ diode laser with a wavelength of $938 \mathrm{~nm}$ and an infrared camera with a resolution of $640 \times 512$ pixels. Different cameras with different frame rates were used: a FLIR A6751sc with $125 \mathrm{~Hz}$, a FLIR X6580sc with $353 \mathrm{~Hz}$ and a FLIR X6901sc at $1003 \mathrm{~Hz}$ (full frame). The laser excitation and the camera are aligned on the same optical axis by a dicroitic mirror (figure 2).

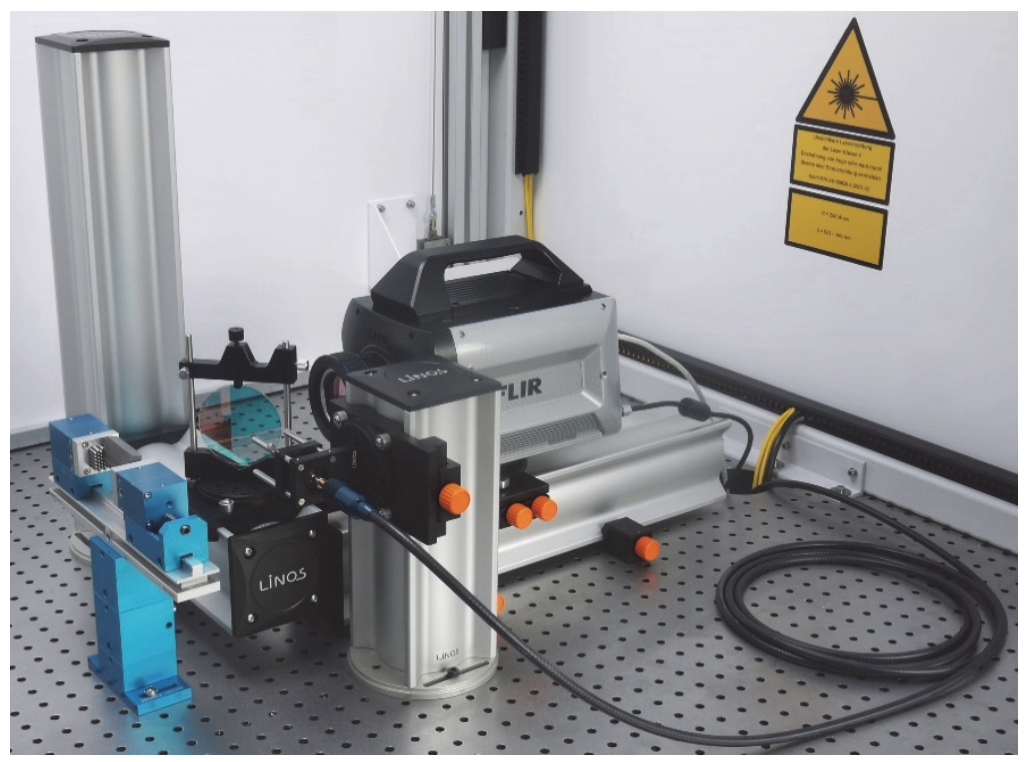

Fig. 2: Lab setup of Forakam: FLIR X6901sc, LTvis 250 NT diode laser fibre, dicroitic mirror and sample in clamping system.

By using an optical table and optical benches, the setup can be changed easily, so different excitation areas can be realized (like spots with 3 and $10 \mathrm{~mm}$ or full-field with $80 \mathrm{~mm}$ diameter). The whole system is included in a laser safety housing with a safety PLC (figure 3 left). Additionally, a more compact sensor head was designed as a demonstrator (figure 3 right).
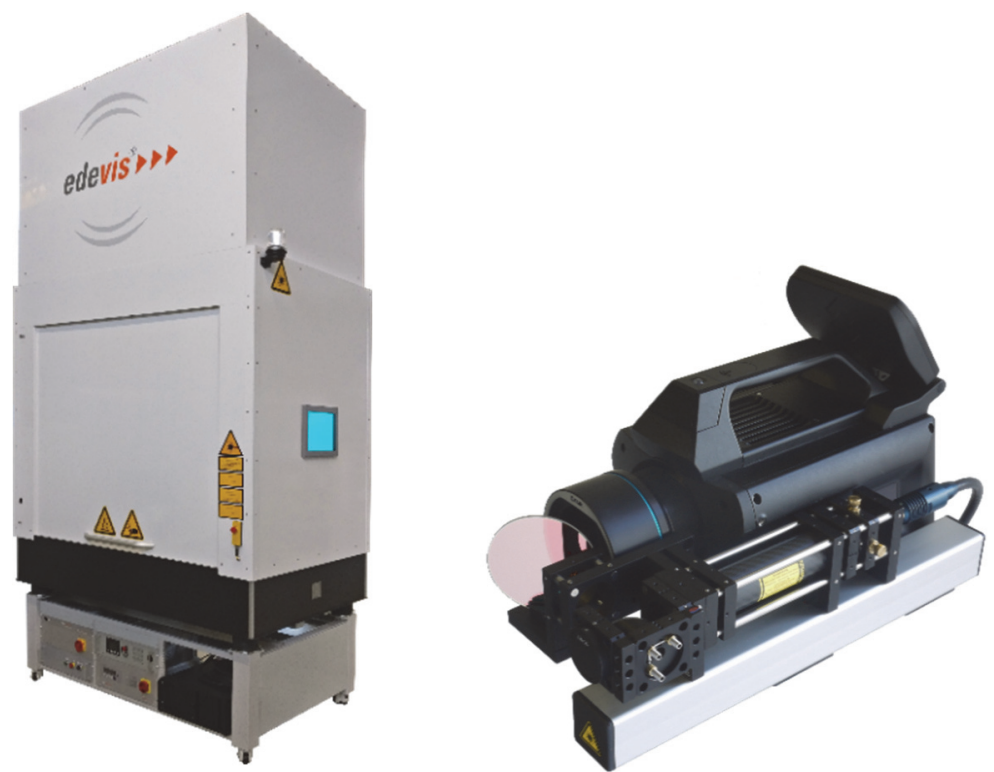

Fig. 3: Industrialized laser cabin (left) and Forakam demonstrator based on FLIR X6580sc (right). 
Two sets of $16 \mathrm{MnCr} 5$ steel samples were tested. One set consisted of six samples with case hardening depths between $0.3 \mathrm{~mm}$ and $2.0 \mathrm{~mm}$ and one non-hardened sample as reference. The second set consisted of four samples with laser-induced artificial grinding burn, manufactured by the IMQ GmbH as a master sample for nital etching training. An identical sample (from the same batch) was used for a metallography analysis by IMQ to determine the extent of the grinding burn.

\section{Results}

\subsection{Case hardening depth measurement}

Case hardening depth measurement is a more challenging task for photothermal radiometry than thickness measurement of a layer on a substrate. There is no exact boundary between two materials with different properties, but there is a gradient from the hardened to the non-hardened material. Additionally, the phase contrast caused by the change of the microstructure is rather small. It is beneficial to use not only one-dimensional heat flow, but also lateral heat flow to increase the SNR. Both effects (the gradient in the material and the 3D heat flow) make a simple fit to an analytical solution impossible.

An example of a Forakam measurement with one excitation spot with a size of about $2 \mathrm{~mm}$ at $32.5 \mathrm{~W}$ is shown in figure 4. The graphs do not show the data of one single pixel, but the median of $7 \times 7$ pixels in the center of the laser spot.

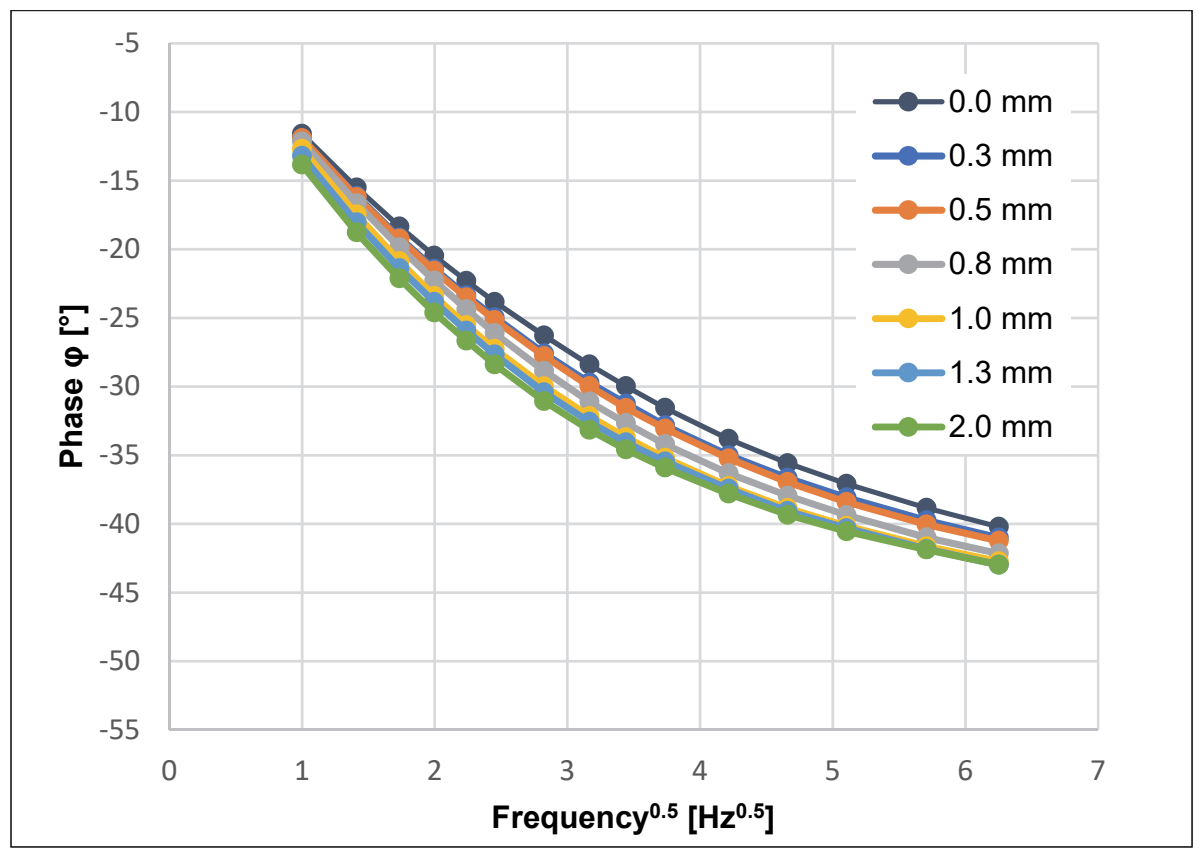

Fig. 4: Phase $\varphi$ of a one spot Forakam measurement of samples with different case-hardening depths.

Still, an estimation of the thickness of the hardened layer is possible by a calibration of the phase values with samples with different case hardening thicknesses. Also, the data is referenced to a non-hardened sample with infinite thermal thickness. So, the evaluation is done not by the absolute phase values, but by the phase contrast. Figure 5 shows the phase contrast of differently hardened samples $(0.3 \mathrm{~mm}$ to $2.0 \mathrm{~mm})$ after subtracting the phase graph of the nonhardened sample $(0.0 \mathrm{~mm})$ from all the other phase graphs. 


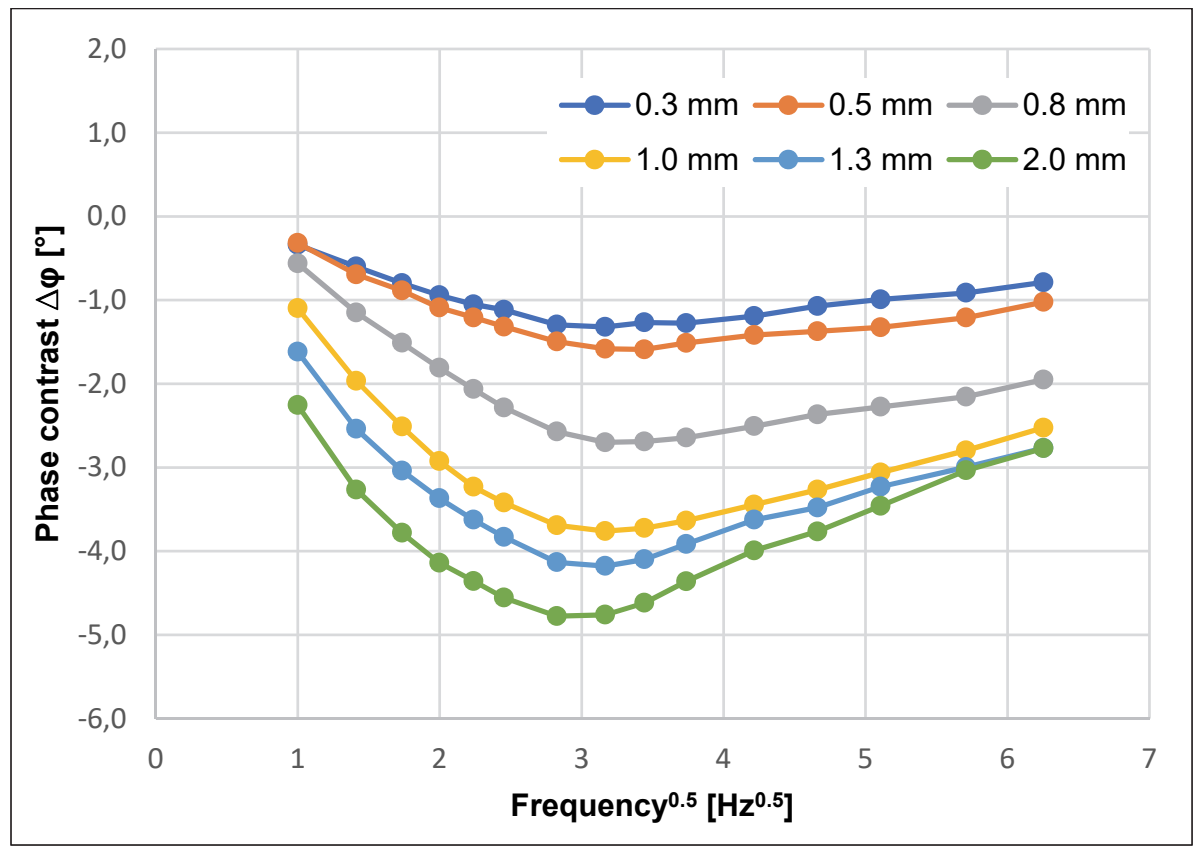

Fig. 5: Phase contrast $\Delta \varphi$ of a one spot Forakam measurement of samples with different case-hardening depths.

This result may be used as a reference for testing components with unknown case hardening depth. Often it is sufficient to determine whether a minimum case hardening depth has been achieved. In this case, only measurements at very few different frequencies are necessary, so the measurement time can be reduced.

For the tests shown above, only a single spot was heated by the modulated laser. In order to be able to do imaging measurements, a diffractive optical element was used to generate a pattern of multiple spots (fig. 6). The DOE - which originally was not designed for the used laser wavelength and power - causes a pattern of one strongly heated center spot, six hexagonally positioned spots with about one third of the amplitude of the center spot, and several additional side maxima with very weak excitation. The overall laser power was $52.5 \mathrm{~W}$.
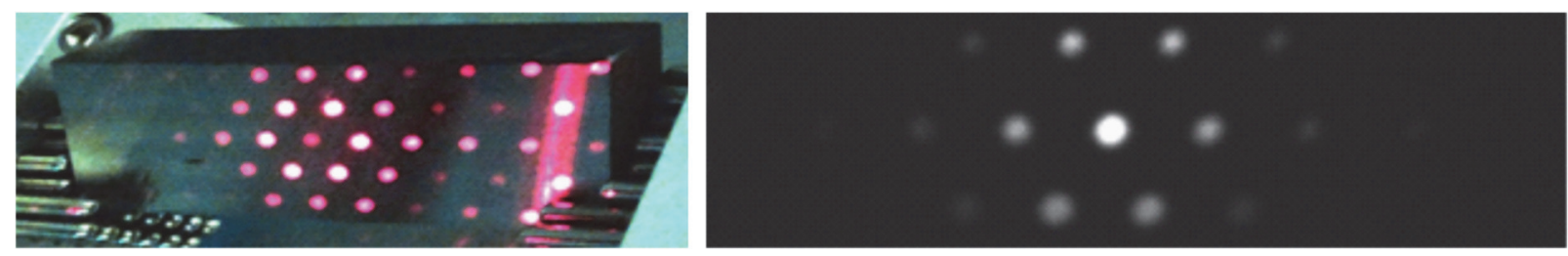

Fig. 6: Multispot laser excitation by a diffractive optical element (left), amplitude image showing the different energy density of the laser spots (right). exemplarily.

By using the DOE, seven spots of a component can be tested at once. Figure 7 shows one phase image

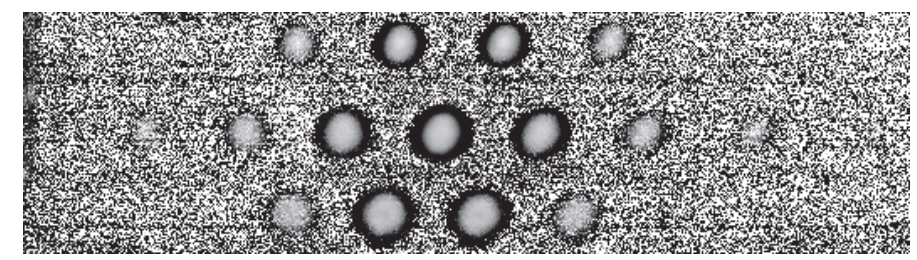

Fig. 7: Phase image of a case-hardened sample, measured with a FLIR X6580sc at $471 \mathrm{~Hz}$ frame rate, $14 \mathrm{~Hz}$ lockin frequency and seven analyzable laser excitation spots.

The phase values shown in figure 8 are the result of a median filtering of the center $7 \times 7$ pixels of the excitation spot in the center of the multispot pattern. 


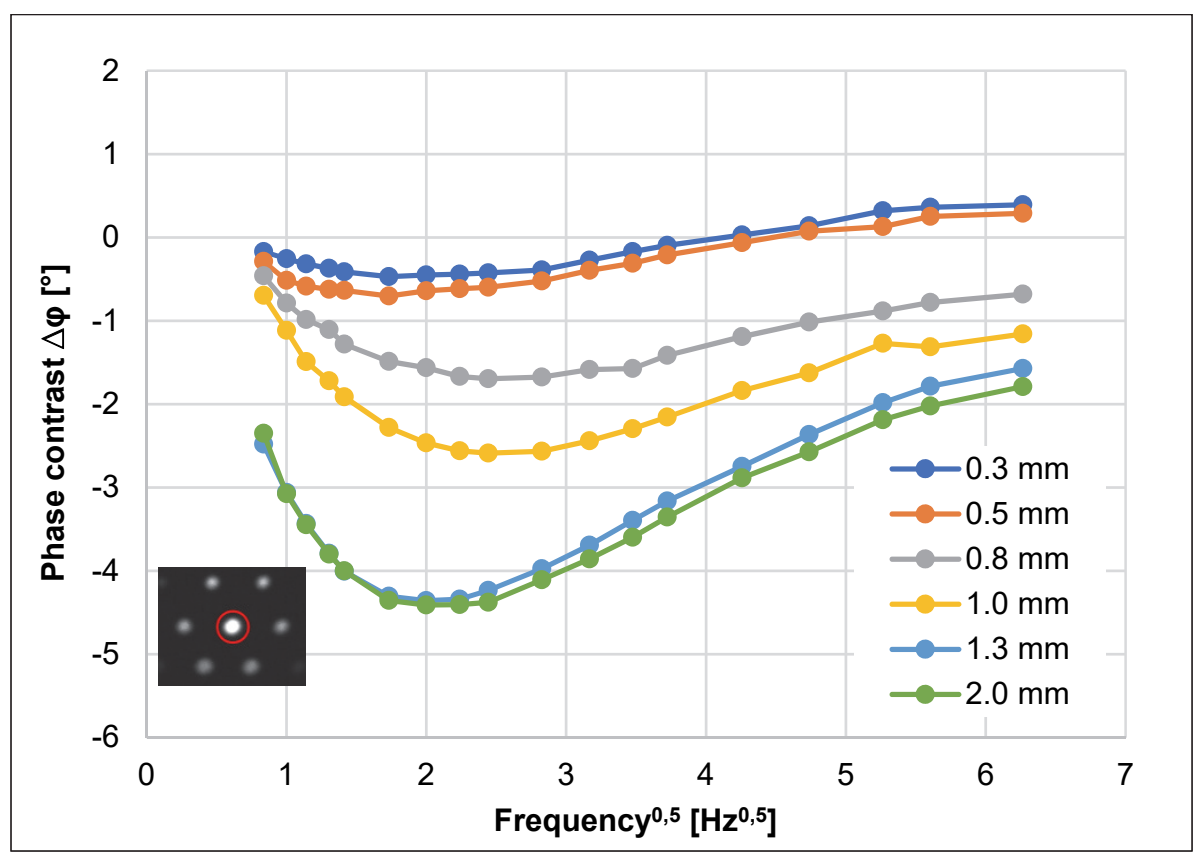

Fig. 8: Phase contrast $\Delta \varphi$ of the center excitation spot of samples with different case-hardening depths.

For comparison, figure 9 shows the median of $7 \times 7$ pixels of the excitation spot on the left side of the multispot pattern.

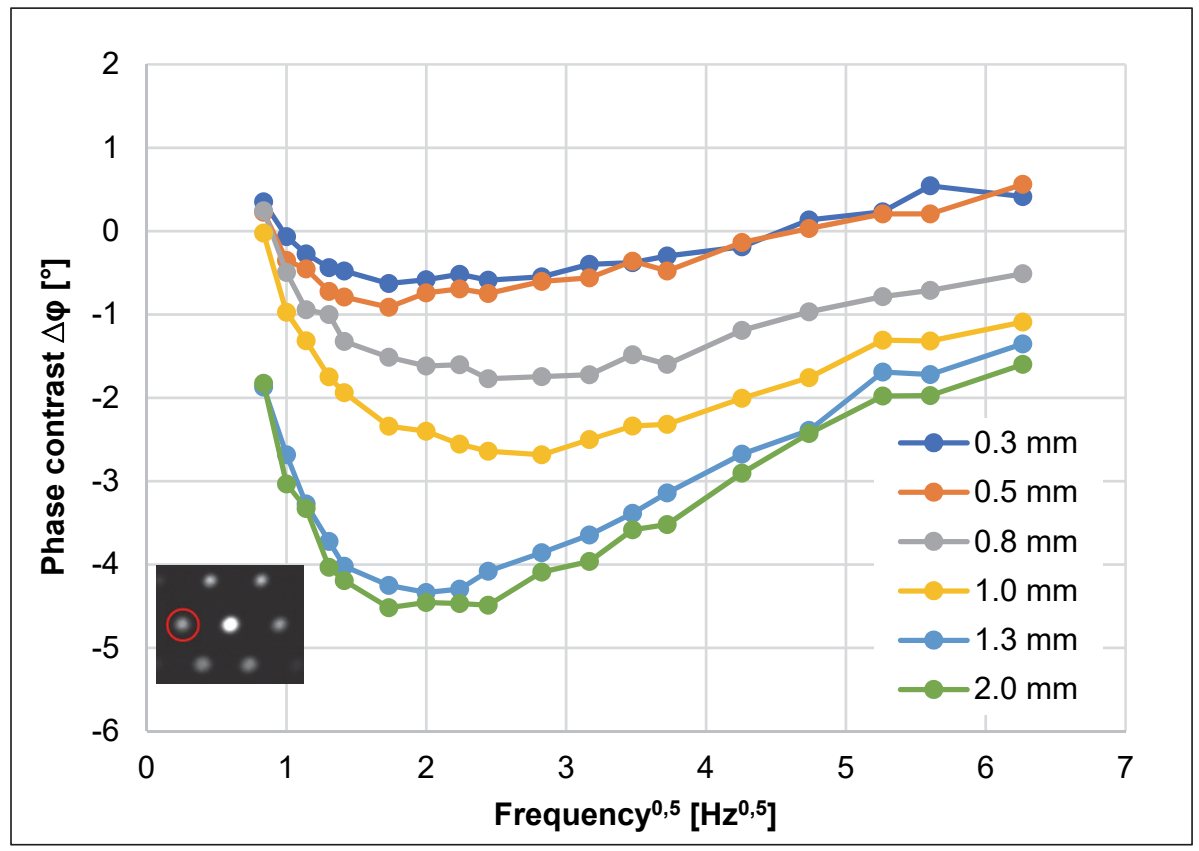

Fig. 9: Phase contrast $\Delta \varphi$ of one of the six hexagonally positioned laser excitation spots of samples with different case-hardening depths.

Figures 8 and 9 are in good agreement. The graphs in figure 9 contain more noise since the laser power in the peripheric six spots was only about one third of the power in the center spot. There are some deviations of both graphs from the graph shown in figure 5 (multispot excitation vs. single spot excitation), especially at low frequencies. Those deviations most likely are caused by a different spot size and potentially a different intensity profile of the spot itself (gaussian vs. top-hat). Those effects influence the phase values by changes of the lateral heat flow. 


\subsection{Grinding burn detection}

Grinding burn also is a heat-affected zone at the surface of a metallic part like case-hardened zones, but it is much thinner than case-hardening, therefore requiring much higher lockin frequencies. The best results were obtained with the FLIR X6901sc at a frame rate of $1003 \mathrm{~Hz}$ (full frame). For the grinding burn studies, reference samples made by the IMQ GmbH were used. The samples contain 10 different heat-affected zones which were generated by a laser. On one of those samples, a metallographic analysis was performed. The result is shown on figure 10.
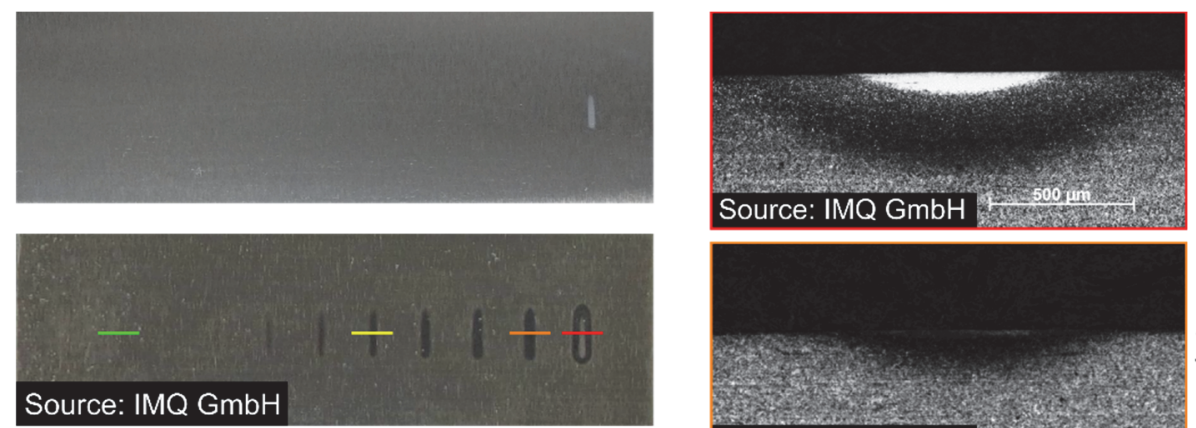

Stage 1:

New-hardening zone $65 \mu \mathrm{m}+/-26$

Tempering zone $350 \mu \mathrm{m}+/-20 \mu \mathrm{r}$

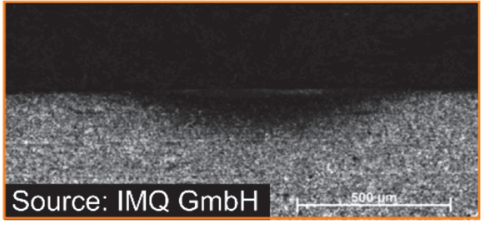

Stage 2:

Tempering zone $200 \mu \mathrm{m}+/-20 \mu \mathrm{n}$

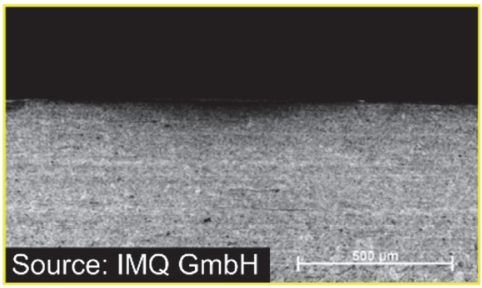

Stage 5

Tempering zone $120 \mu \mathrm{m}+/-20 \mu$

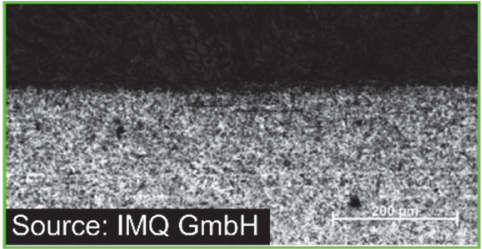

Stage 10:

Tempering zone $25 \mu \mathrm{m}+/-20 \mu \mathrm{m}$

Fig. 10: Grinding burn reference sample before etching (top left) and after etching (bottom left), metallographic analysis showing new-hardening zone and tempering zones with different thicknesses (right).

After etching, nine tempering zones and one combination of a new hardening zone and a tempering zone are visible. On another (non-etched) sample, Forakam was used to detect the simulated grinding burn in an imaging way without any chemical treatment. The result of the completely non-destructive inspection at $105 \mathrm{~Hz}$ is shown in figure 11 .
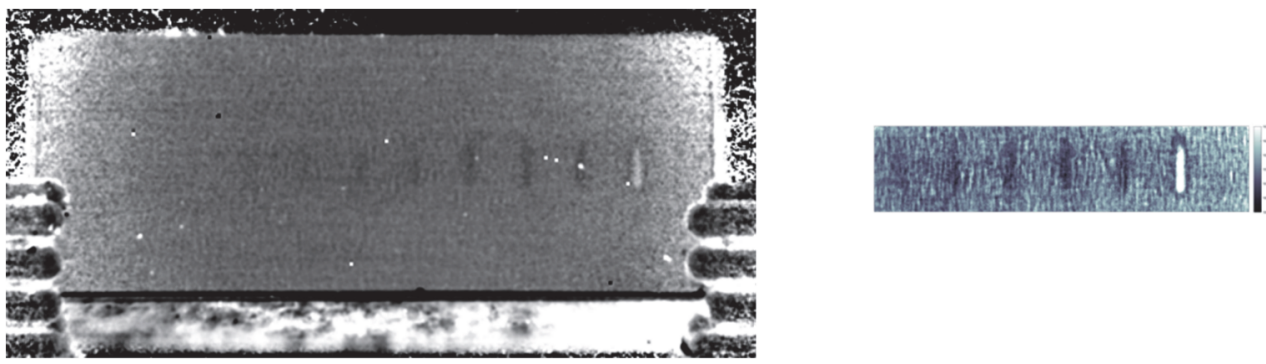

Fig. 11: Phase images of a grinding burn reference sample, tested at $105 \mathrm{~Hz}$ lockin frequency with imaging Forakam (left) and scanning Foratherm (right).

With Forakam, tempered zones up to stage 6 (corresponding to a thickness of about $100 \mu \mathrm{m}$ ) could be detected within $25 \mathrm{~s}$ in a field of view of about $55 \times 25 \mathrm{~mm}^{2}$. With the scanning Foratherm system, the inner part of the sample was scanned with the modulated laser spot. The results have a better signal-/noise-ratio than with Forakam. However, the Inspection took more than 1 hour. After fully implementing the camera interface of the FLIR 6901sc, Forakam could not only use the internal camera SSD (which limited the recording time to $26 \mathrm{~s}$ ), but a basically infinite recording time could be used. Figure 12 shows the result which could be obtained with $200 \mathrm{~s}$ inspection time. 


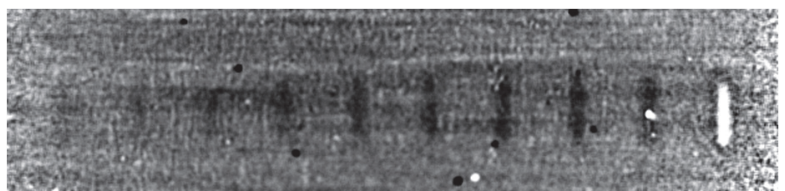

Fig. 12: Phase image $(315 \mathrm{~Hz})$ of a grinding burn reference sample, tested with FLIR X6901sc at $1260 \mathrm{~Hz}$ frame rate, 640 x 140 px subwindow and 315 Hz lockin frequency.

At $315 \mathrm{~Hz}$ lockin frequency and an inspection time of $200 \mathrm{~s}$, all simulated grinding burn areas could be detected. The SNR of this Forakam result is about the same as with Foratherm, while the inspection time could be reduced by the number of pixels of the IR camera which were used (in this case $89600 \mathrm{px}$ ). An automated detection should be possible up to stage 6 , which is a tempering zone with a thickness of about $100 \mu \mathrm{m}$.

\section{Conclusion}

It was demonstrated that photothermal radiometry can be performed in an imaging way. Especially the grinding burn detection on larger parts can be quickened by several orders of magnitude. Quantitative values of the thickness of layers on substrates are accessible through the analytic solution for 1D heat flux and material parameters. Case-hardening depths can be estimated after a calibration with reference parts with known case hardening depths by evaluating the phase contrast.

\section{Acknowledgements}

The authors would like to thank the Federal Ministry for Economic Affairs and Energy (BMWi) for funding the Forakam project (KF2201505GR4) as part of the Central Innovation Program (ZIM).

\section{REFERENCES}

[1] Nordal P E, Kanstad S O Photothermal Radiometry. Physica Scripta 1979;20:659-662

[2] Busse G, Optoacoustic phase angle measurement for probing a metal. Applied Physics Letters 1979:35:759760

[3] Bennett C A, Patty R R Thermal wave interferometry: a potential application of the photoacoustic effect. Applied Optics 1982;21:49-54

[4] Lindemann S, Schaller R Laser-angeregte Lockin-Thermografie zur zerstörungsfreien und berührungslosen Schichtdickenmessung von thermisch gespritzen Beschichtungen. Proceedings of DGZfP Annual Meeting 2017, Koblenz

[5] Vergleichskörper NE Test Set: Gebrauchsmuster Nr. 202015001 664.2, IPC G01N 27/72, German Patent Office Munich

[6] Seidel M, Zösch A, Härtel K Neu entwickeltes Test-Set für die Überwachung der Schleifbrandätzung. Schleifen und Polieren 2016;1:32-34 